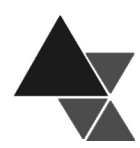

\title{
Comparando a alimentação dentro e fora do domicílio, no Brasil, em 2008-2009 1
}

\author{
Rodolfo Hoffmann ${ }^{2}$
}

Alguns artigos sugerem que a alimentação fora do domicilio, no Brasil, é menos saudável que a consumida no domicílio, associando a primeira com fast food. A análise dos dados do inquérito alimentar da Pesquisa de Orçamentos Familiares (POF) 2008-2009 não sustenta essa ideia. É usual comparar a alimentação de quem eventualmente come fora do domicilio $(40,2 \%)$ com a de quem se alimenta somente no domicílio (59,8\%). Isso implica comparar grupos de pessoas muito diferentes. Os que eventualmente comem fora do domicilio têm maior renda per capita (45\% maior) e escolaridade ( 8,8 versus 6,4 anos), são mais jovens (32,1 versus 40,5 anos) e têm maior ingestão calórica diária (2.063 kcal versus $1.801 \mathrm{kcal})$. Para estimar o efeito de "comer fora" é necessário controlar o efeito de outros fatores, incluindo alguns não observáveis. Usando os dados referentes às pessoas que comem tanto dentro como fora do domicílio (amostra de 12.045 pessoas), foi estimado o efeito de "comer fora" controlando o efeito de todas as características pessoais. Essa estimativa é obtida utilizando, para cada pessoa a diferença, no valor da variável dependente, entre alimentação fora e dentro do domicilio. Verifica-se que, para cada $100 \mathrm{kcal}$ de alimentos, a alimentação fora do domicilio apresenta menos sal e mais frutas e vitamina C. Por outro lado, a alimentação fora do domicílio apresenta maior teor de açúcar e menor teor de vitamina A. Mostra-se, também, que o fato de comer fora do domicílio está associado a uma menor probabilidade de uma mulher apresentar sobrepeso ou obesidade.

Palavras-chave: alimentação fora do domicílio, dados pareados, sobrepeso, obesidade.

\section{Comparing the quality of eating out and eating at home in Brazil}

Some papers suggest that out-of-home eating in Brazil is less healthy than food consumed at home, linking the former to junk food. Such view is mistaken, as emerges from an analysis of data from the Brazilian Nationwide Dietary Survey conducted along with the 2008-2009 Household Budget Survey (POF 2008-2009). Usually the comparison is between those who eventually eat out $(40.2 \%)$ and those who eat only at home $(59.8 \%)$. This means comparing very different groups of people. Those who eventually eat out on average have a higher family income per capita (45\% higher) and schooling (8.8 years as compared to 6.4), are younger (32.1 years old versus 40.5) and their daily calories consumption is higher $(2,063 \mathrm{kcal}$ versus 1,801$)$. In order to estimate the impact of "eating out" on diet quality it is necessary to control for the effects of other factors, in particular income levels. Such control is not always rigorous, and unobserved variables such as gluttony might also have an impact. Using the information about people eating out or at home (sample of 12,045 persons) we estimate the impact of "out-of-home eating" controlling for all the individual characteristics, even gluttony. An appropriate estimate is obtained by considering, for each individual, the difference in the value of the dependent variable between eating outside home and eating at home. We show that, for each $100 \mathrm{kcal}$ of food consumed away from home fat and salt content is lower, and fruit and $\mathrm{C}$ vitamin content is higher. On the other hand, food outside home presents lower A vitamin and iron content and more sugar. It is also shown that away-from-home eating is associated with a lower probability of a woman presenting overweight or obesity.

\footnotetext{
${ }^{1}$ Os resultados mais importantes dessa pesquisa foram apresentados no 20 Congresso Internacional de Nutrição, Granada (Espanha), 15 a 20 de setembro de 2013.

2 Professor Sênior da Escola Superior de Agricultura "Luiz de Queiroz" (ESALQ), Universidade de São Paulo (USP), com apoio do Conselho Nacional de Desenvolvimento Científico e Tecnológico (CNPq).E-mail: hoffmannr@usp.br.
} 
Key-words: away-from-home eating, paired data, overweight, obesity, Brazil.

\section{INTRODUÇÃO}

Será que a alimentação consumida fora do domicilio, como um todo, pode ser considerada menos saudável que aquela ingerida no domicílio? A análise dos dados sobre consumo pessoal de alimentos da Pesquisa de Orçamentos Familiares (POF) de 20082009, com uma amostra representativa de todo o Brasil, permite esclarecer aspectos importantes dessa questão. Utilizando os dados referentes a pessoas que comem tanto dentro como fora do domicílio, é possível comparar várias características desses dois tipos de alimentação controlando o efeito de todas as características da pessoa.

Alguns trabalhos têm indicado que no Brasil, da mesma maneira que em diversos outros países, a alimentação fora do domićlilio é menos saudável do que a alimentação ingerida no domicílio [1,2,3]. Veremos que parte das conclusões desses trabalhos não é confirmada por uma análise mais cuidadosa dos dados da POF 2008-2009'.

Outros trabalhos assinalam, cautelosamente, que a alimentação fora do domicílio não é necessariamente de pior qualidade, particularmente no caso dos restaurantes "por quilo" [5,6].

\section{METODOLOGIA}

Os dados da POF de 2008-2009 foram coletados pelo Instituto Brasileiro de Geografia e Estatística (IBGE) de 19 de maio de 2008 a 18 de maio de 2009. A amostra é constituída por 55.970 domicilios particulares permanentes em áreas rurais e urbanas do Brasil. Para uma subamostra de 13.569 domicílios foram obtidas informações sobre o consumo alimentar pessoal para todos os seus moradores com 10 anos ou mais de idade (34.003 moradores) [7]. Para cada pessoa do inquérito alimentar o IBGE fornece um fator de

${ }^{1}$ Este artigo já estava redigido quando tomamos conhecimento do trabalho de Bezerra et al. (2013) [4] também baseado nos dados da POF 2008-2009. Há concordância em constatar que o percentual de pessoas que consomem alimentos fora do domicílio diminui com a idade, aumenta com a renda e é maior entre homens e na área urbana. Mas a afirmativa de que "a alimentação fora de casa apresenta [...] pobre conteúdo nutricional” não nos parece correta. Essa afirmativa não é devidamente suportada pela análise desenvolvida no trabalho das autoras e, em certos aspectos, é negada pelos resultados apresentados no presente artigo. expansão que corresponde ao número de pessoas da população representadas por aquela pessoa da amostra. Todas as análises estatísticas realizadas para esse trabalho foram ponderadas por esse fator de expansão.

Cada morador, com 10 anos ou mais de idade, registrou em dois dias não consecutivos, seu consumo individual. [...] O método de registro alimentar apresenta a vantagem de ser independente da memória, uma vez que os alimentos são anotados no momento do consumo (IBGE, 2011, p. 30) [7].

Da mesma maneira que foi feito pelo próprio IBGE, utilizamos os dados do primeiro dia de registro [7].

Para cada item foi registrado se o consumo ocorria "dentro do domicílio" ou "fora do domicilio".

O cálculo do consumo de energia, proteína, carboidrato, lipídeos, vitamina $\mathrm{C}$ etc. foi feito utilizando a tabela de conversão fornecida pelo IBGE [8].

Para comparar a alimentação consumida dentro e fora do domicilio, uma alternativa é separar as pessoas que comem apenas no domicilio daquelas que ingeriram alimentos fora do domicílio. É claro que isso envolve a comparação da alimentação de pessoas que são diferentes em outros aspectos. Veremos, por exemplo, que os que comem fora do domicílio apresentam maiores médias de renda per capita e escolaridade. Pode-se tentar controlar o efeito dessas variáveis por meio de regressões múltiplas, mas o controle nunca é perfeito, pois não conhecemos a forma matemática da relação e pode haver influência substancial de uma variável não observada. Apenas para esclarecer a questão estatística, vamos denominar de gula as variações entre pessoas em certas características relativas ao consumo de alimentos (procurando esquecer o aspecto moral associado ao fato de a gula ser considerada um dos 7 pecados capitais). É razoável pressupor que a dieta varia com a gula e que a probabilidade de comer fora do domicílio é maior para os gulosos. Assim, ao comparar a dieta dos que comem fora do domicílio com a dos que comem apenas no domicílio, há o risco de atribuir ao fato de 
comer fora diferenças que são, na realidade, determinadas por características diferentes das pessoas (mesmo controlando cuidadosamente as características observáveis como renda, escolaridade, sexo e idade).

Considerando apenas as pessoas que comem tanto dentro como fora do domicílio, é possível usar os dados do inquérito alimentar da POF 2008-2009 para comparar os dois tipos de alimentação controlando todas as características pessoais, inclusive as não observadas. Trata-se de uma aplicação do teste $t$ para dados pareados, podendo-se utilizar, para cada pessoa, a diferença entre os valores do indicador de qualidade (y) para a alimentação fora e dentro do domicílio.

Foram utilizados vários indicadores (y) para avaliar a qualidade da dieta. Três deles são as porcentagens de calorias provenientes de proteínas, carboidratos e lipídeos, admitindo que cada grama desses nutrientes corresponda, respectivamente, a 4, 4 e 9 quilocalorias.

A maioria dos indicadores consiste na quantidade de um elemento de interesse (alimento, fibra, vitamina $\mathrm{C}$ etc.) para cada $100 \mathrm{kcal}$ de alimentos ingeridos. Assim, denominamos de teor de vitamina $\mathrm{C}$ de determinado tipo de alimentação a quantidade média de vitamina C (mg) por $100 \mathrm{kcal}$ de alimento.

\section{RESULTADOS E DISCUSSÃO}

Analisando os microdados da POF 2008-2009, inicialmente foram reproduzidos alguns resultados já apresentados na publicação do IBGE [7]. Assim, considerando a amostra de 34.003 pessoas do inquérito alimentar, verifica-se que na população correspondente (160,5 milhões de pessoas) 53,9\% são do sexo feminino, 83,5\% residem em área urbana e para $40,2 \%$ ocorre ingestão de energia fora do domicilio ${ }^{2}$. Da ingestão calórica total, $16,2 \%$ é proveniente da alimentação fora do domicílio. O consumo médio diário de feijão é 182,9 g por pessoa, sendo $12,2 \%$ consumido fora do domicílio. A publicação do IBGE apresenta esse tipo de informação para uma longa lista de alimentos.

\footnotetext{
${ }^{2}$ Há, na amostra, um homem (do RS, com 377 meses de idade e 11 anos de escolaridade) cujo consumo fora do domicílio consistiu apenas em $300 \mathrm{~g}$ de refrigerante de guaraná diet. Como não houve consumo de energia fora do domicílio, optamos por classificar essa pessoa no grupo dos que não come fora. Dessa maneira, a amostra fica com 21.462 pessoas que não comem fora e 12.541 que comem fora, isto é, que ingerem, fora do domicílio, alimentos com conteúdo energético positivo.
}

A alimentação fora do domicílio contribui com $20,7 \%$ do consumo de alface (mais do que os 16,2\% de participação na ingestão calórica total). Mas a participação da alimentação fora do domicílio é particularmente elevada para vários alimentos considerados indicadores de má qualidade da dieta: cerca de $40 \%$ dos refrigerantes, mais de $50 \%$ dos salgados e salgadinhos industrializados e 63,6\% da cerveja. Isso não deve ser associado, exclusivamente, a diferenças entre os dois tipos de alimentação. É necessário considerar que as pessoas que comem fora do domicilio têm, em média, características muito diferentes das que comem exclusivamente no domicílio.

\section{Fatores associados com a probabilidade de uma pessoa comer fora do domicílio}

Uma vez que entre os fatores que afetam a probabilidade de uma pessoa comer fora do domicílio será considerada a escolaridade e a cor da pessoa, é necessário excluir da amostra as pessoas sem declaração para essas variáveis. Optou-se por excluir, também, os indígenas. Restou uma amostra de 33.453 pessoas, representando uma população de 158,2 milhões.

Entre os residentes em áreas urbanas, 42,7\% comem fora do domicílio, mas para os residentes em áreas rurais essa porcentagem é $27,2 \%$. A proporção de pessoas que come fora é menor para o sexo feminino: $36,3 \%$, contra $44,3 \%$ para o sexo masculino. Para brancos, amarelos, pretos e pardos a proporção que come fora do domicilio é 43,1\%, 39,3\%, 37,8\% e $37,3 \%$, respectivamente.

Mas será que há um efeito específico da cor ou a probabilidade de comerem fora do domicílio é menor para pretos e pardos porque sua renda per capita média é menor? Para estimar o efeito de cada variável controlando os efeitos das demais, foram ajustados os modelos de lógite apresentados na Tabela 1.

Foi estimado um modelo utilizando todas as observações disponíveis (33.453), um considerando apenas mulheres adultas (14.186 observações) e outro apenas para homens adultos (11.753 observações).

Excluindo a idade, todas as variáveis explanatórias são variáveis binárias, assumindo valor 1 para determinada categoria e valor 0 nas demais. Foram utilizadas 7 variáveis binárias para distinguir 8 níveis de escolaridade, adotando como base o estrato com 
escolaridade inferior a 1 ano. Entre regiões a base é o Nordeste e para cor considera-se como base os brancos. Foram definidos 10 estratos de Renda Familiar Per Capita (RFPC), delimitados em reais de janeiro de 2009, adotando-se como base o grupo cuja RFPC não supera R\$ 140 por mês. A variável "Urbano" permite captar o efeito da residência urbana, em comparação com a rural. Outra variável permite verificar se existe um efeito adicional associado à residência em área metropolitana. São usadas 2 variáveis binárias para distinguir 3 situações no que refere ao número de crianças de 0 a 4 anos na família: nenhuma, uma e duas ou mais. Procedimento análogo é utilizado para captar o efeito da presença de crianças de 5 a 9 anos.

No modelo para pessoas com 10 ou mais anos de idade observa-se que a probabilidade de comer fora é significativamente mais baixa para as do sexo feminino. O efeito da idade foi razoavelmente bem captado por meio de um polinômio do 4으 grau. A Figura mostra como a probabilidade estimada de comer fora varia com a idade, considerando uma pessoa branca do sexo masculino, com 5 a 8 anos de escolaridade, em área urbana do Nordeste, com RFPC no estrato de mais de $R \$ 280$ a $R \$ 560$ e sem crianças de até 9 anos de idade na família.

A probabilidade de comer fora do domicílio é especialmente alta para crianças de 10-11 anos porque é muito provável que ela esteja frequentando escola e consumindo a merenda escolar. Sabemos que a proporção de alunos que come a merenda, em escolas públicas de $1 \stackrel{0}{\text { grau}}$, cai substancialmente com a idade [9]. Isso permite compreender, na Figura, a redução da probabilidade de comer fora na faixa de 10 a 15 anos de idade. Em seguida essa probabilidade foi relativamente estável, voltando a diminuir mais intensamente a partir da faixa de 40-45 anos de idade.

Nos modelos ajustados para mulheres ou homens com 20 ou mais anos de idade, ainda foi necessário usar um polinômio do 3 o grau para captar apropriadamente o efeito da idade, pois as curvas apresentam um ponto de inflexão próximo aos 60 anos de idade. É claro que considerando apenas o efeito linear obtém-se um controle insatisfatório do efeito da idade.

Nos 3 modelos ajustados observa-se que a probabilidade de comer fora tende a crescer com a escolaridade e com a RFPC e é maior nas áreas urbanas do que nas rurais. O efeito da RFPC é mais intenso no caso dos homens.

No caso dos homens, a residência em área metropolitana tem um efeito positivo adicional ao efeito da variável "Urbano".

Como esperado, a presença de uma criança de 0 a 4 anos de idade na família têm efeito negativo sobre a probabilidade de uma mulher comer fora, mas, curiosamente, o efeito é positivo no caso dos homens.

\section{Diferenças entre alimentação dentro e fora do domicílio}

Nesta seção procura-se estabelecer a relação entre "comer fora do domicilio" e a qualidade da dieta ingerida. Para isso voltamos a considerar a amostra completa do inquérito alimentar da POF 2008-2009, com 34.003 pessoas.

Para facilitar a apresentação dos resultados, vamos definir 3 grupos de pessoas na amostra analisada:

Grupo A: pessoas sem ingestão de energia fora do domićlio; inclui 21.451 pessoas da amostra de 34.003 pessoas do inquérito alimentar.

Grupo B: pessoas com ingestão de energia fora do domicílio; inclui as demais 12.542 pessoas da amostra.

Grupo C: pessoas com ingestão de energia tanto dentro como fora do domicílio; inclui 12.045 pessoas da amostra, que são parte do Grupo B, pois há 497 pessoas com ingestão de energia exclusivamente fora do domicílio.

Podemos avaliar o efeito de "comer fora do domicílio" comparando os grupos A e B, mas isso envolve comparar dietas de pessoas muito diferentes em vários aspectos, como mostra a Tabela 2.

Para obter uma estimativa do efeito de "comer fora" com controle de todas as características intrínsecas à pessoa (inclusive suas preferências alimentares), é interessante comparar as dietas da alimentação "dentro" e "fora" para as pessoas do grupo C. 
Tabela 1. Modelos de lógite estimados para avaliar os condicionantes da probabilidade de uma pessoa comer fora, no Brasil, 2008-2009

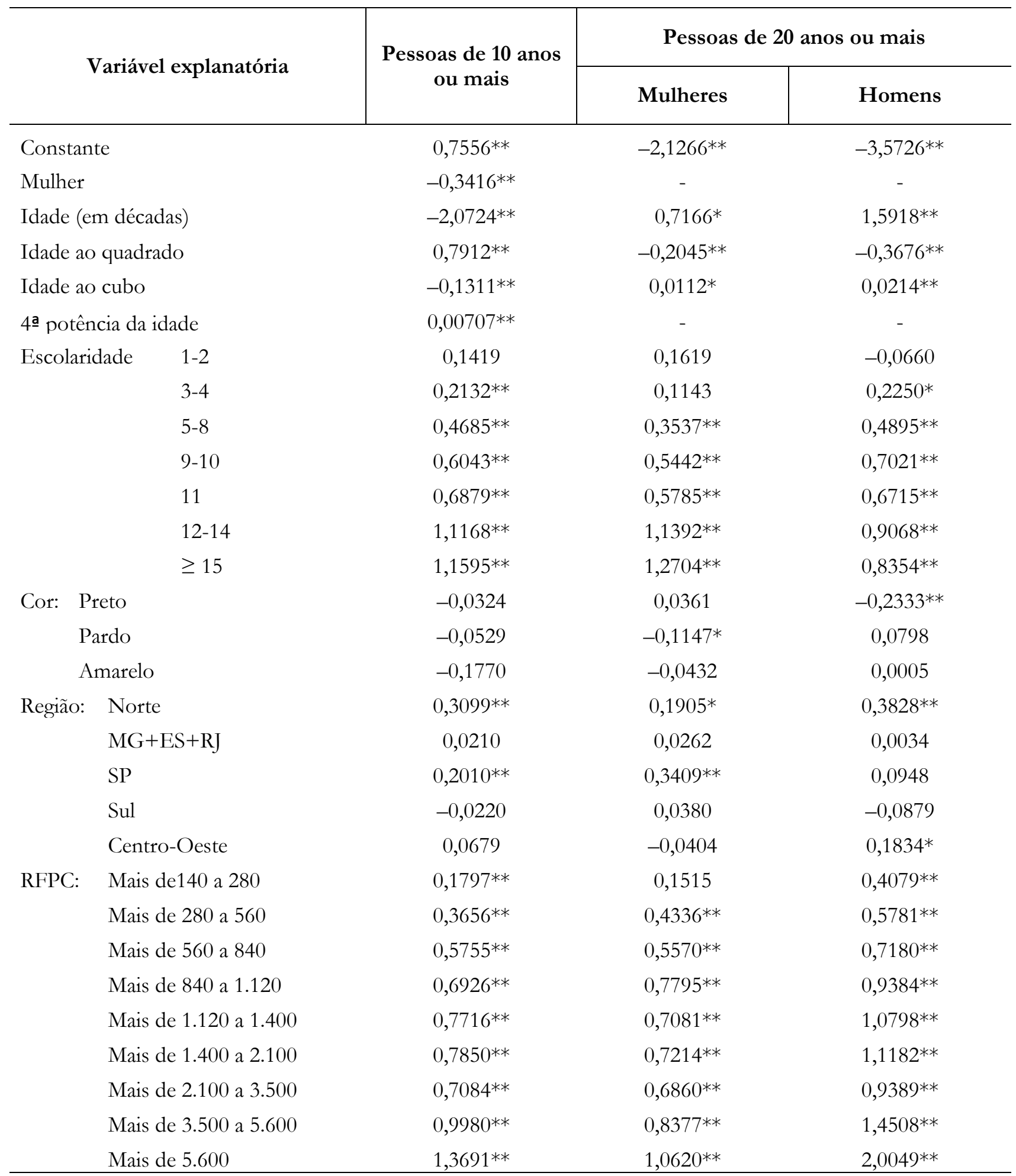


Tabela 1. Continuação

\begin{tabular}{|c|c|c|c|}
\hline \multirow{2}{*}{ Variável explanatória } & \multirow{2}{*}{$\begin{array}{l}\text { Pessoas de } 10 \text { anos } \\
\text { ou mais }\end{array}$} & \multicolumn{2}{|c|}{ Pessoas de 20 anos ou mais } \\
\hline & & Mulheres & Homens \\
\hline Urbano & $0,3325^{* *}$ & $0,4312^{* *}$ & $0,3509 * *$ \\
\hline Metropolitano & 0,0324 & $-0,0463$ & $0,2669 * *$ \\
\hline \multirow[t]{2}{*}{ Criança de 0 a 4 anos: } & $-0,1209 * *$ & $-0,3667 * *$ & $0,1620^{* *}$ \\
\hline & $-0,2062^{* *}$ & $-0,7624 * *$ & 0,0867 \\
\hline \multirow[t]{2}{*}{ Criança de 5 a 9 anos: } & 0,0048 & $-0,1465^{* *}$ & $0,1464^{*}$ \\
\hline & $-0,1895^{* *}$ & $-0,1991$ & $-0,3161 *$ \\
\hline$\chi^{2}$ razão de verossimilhança & $4.638,2^{* *}$ & $2.292,6^{* *}$ & $1.867,1^{* *}$ \\
\hline Medida de concordância $c$ & 0,707 & 0,731 & 0,716 \\
\hline Número de observações & 33.453 & 14.186 & 11.753 \\
\hline
\end{tabular}

Figura. Variação da probabilidade estimada de comer fora em função da idade

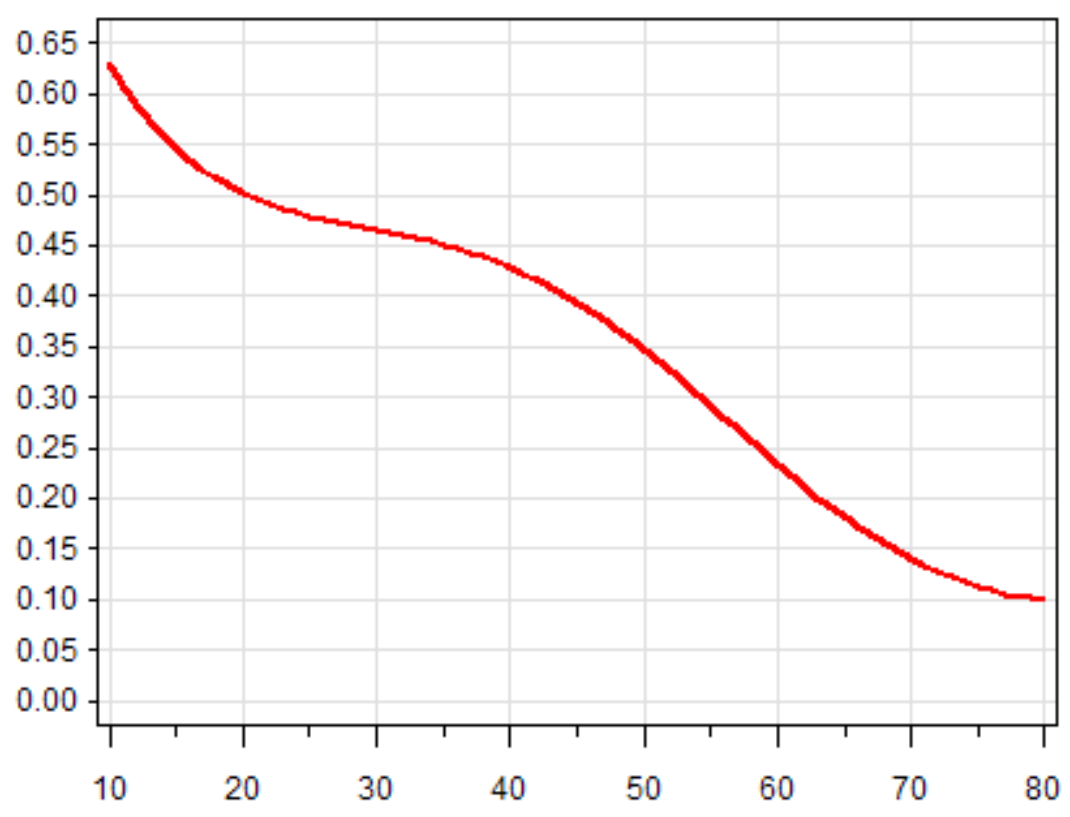

Idade (em anos) 
Tabela 2. Características dos grupos A (só comem no domićlio), B (comem fora do domicílio) e C (comem tanto dentro como fora do domicílio)

\begin{tabular}{lccc}
\multicolumn{1}{c|}{ Variável } & Grupo A & Grupo B & Grupo C \\
\hline Proporção feminina (\%) & 55,1 & 46,7 & 47,0 \\
Proporção de brancos (\%) & 46,1 & 52,0 & 52,2 \\
Proporção de pretos (\%) & 8,5 & 7,7 & 7,7 \\
Proporção de pardos (\%) & 44,0 & 39,0 & 38,7 \\
Proporção urbana (\%) & 79,9 & 88,8 & 88,9 \\
Idade média & 40,5 & 32,1 & 31,9 \\
Média da RFPC (R\$) & 761 & 1.106 & 1.104 \\
Escolaridade média (1) & 6,4 & 8,8 & 8,8 \\
\hline
\end{tabular}

(1) Exclusive 216 observações sem declaração de escolaridade, e atribuindo valor 17 para os com 15 anos ou mais de estudo.

Seja $x$ o consumo diário de algum alimento ou componente de alimentos. Assim, conforme o elemento considerado, $x_{A}$ pode representar o consumo diário de feijão, de lipídeos ou de vitamina $\mathrm{C}$ de uma pessoa do grupo A. Para uma pessoa do Grupo B as mesmas variáveis seriam representadas por $x_{B}$.

Vamos indicar por $y$ o teor de $x$ por 100 quilocalorias. Tratando-se de vitamina $\mathrm{C}$, por exemplo, $y_{A}$ seria a quantidade de vitamina $C$ para cada $100 \mathrm{kcal}$ de alimentos consumidos por pessoa do grupo A. Para proteína, carboidratos, açúcar e lipídeos, $y$ representa a porcentagem de calorias proveniente de cada um desses nutrientes.

Ao comparar a alimentação dentro e fora do domicílio nas pessoas do grupo $\mathrm{C}$, os teores da alimentação dentro e fora do domicílio para cada pessoa são indicados por $y_{1}$ e $y_{2}$, respectivamente.

A ingestão calórica diária média é igual a 1.801 kcal no grupo A e 2.063 no grupo B. No grupo C essa média é $2.068 \mathrm{kcal}$, sendo $1.344 \mathrm{kcal}$ provenientes da alimentação no domicilio e $724 \mathrm{kcal}$ provenientes da alimentação fora do domicilio.

A Tabela 3 mostra os principais resultados.
Da mesma maneira que a ingestão energética total, a ingestão média de lipídeos também é maior no grupo B do que no grupo A. O teor de lipídeos, isto é, a participação dos lipídeos no total de energia da alimentação também é maior no grupo $\mathrm{B}$ do que no grupo A. Entretanto, quando se controla o efeito de todas as características individuais, verifica-se que o teor de lipídeos é estatisticamente menor no consumo de alimentos fora do domicílio. $\mathrm{O}$ fato de, no caso dos lipídeos, termos, em média, $x_{B}>x_{A}$ e $y_{B}>y_{A}$ não se deve a uma característica intrínseca à alimentação fora do domicílio, mas às características diferentes dos que comem eventualmente fora do domicílio, em comparação aos que comem apenas no domicílio, cabendo destacar o nível mais elevado de renda dos primeiros.

Merecem destaque, também, os resultados referentes ao sódio e ao colesterol. O consumo médio é maior no grupo $\mathrm{B}$ do que no grupo $\mathrm{A}$, mas o teor é menor no grupo B. E quando são controlados os efeitos das características pessoais, verifica-se que o teor é menor no consumo fora do domicilio.

O fato de, dentro do grupo $\mathrm{C}$, o teor de vitamina $\mathrm{C}$ ser maior na alimentação fora do domicílio também contradiz a ideia de que esta seja menos saudável. 
Tabela 3. Três formas de comparar a alimentação dentro e fora do domicílio no Brasil, com base na Pesquisa de Orçamentos Familiares (POF) 2008-2009

\begin{tabular}{|c|c|c|c|}
\hline \multirow{2}{*}{ Item } & \multicolumn{2}{|c|}{ Comparação de médias nos Grupos A e B } & \multirow{2}{*}{$\begin{array}{c}\text { Média das diferenças } y_{2}-y_{1} \\
\text { no grupo } \mathrm{C}\end{array}$} \\
\hline & $\bar{x}_{B}-\bar{x}_{A}$ & $\bar{y}_{B}-\bar{y}_{A}$ & \\
\hline Proteína & $+6,38^{* *}$ & $-0,79 * *$ & $-0,528^{* *}$ \\
\hline Carboidrato & $+33,6^{* *}$ & $-0,65^{* *}$ & $+1,06^{* *}$ \\
\hline Lipídeos & $+9,90^{* *}$ & $+1,00^{* *}$ & $-0,221^{* *}$ \\
\hline Açúcar & $+26,7^{* *}$ & $+3,03^{* *}$ & $+2,29 * *$ \\
\hline Arroz & $-9,45^{* *}$ & $-1,66^{* *}$ & $-2,95^{* *}$ \\
\hline Feijão & $-16,28^{* *}$ & $-2,15^{* *}$ & $-3,32^{* *}$ \\
\hline Alface & $+1,35^{* *}$ & $+0,038^{* *}$ & $-0,0042$ \\
\hline Tomate & $+0,90^{* *}$ & $+0,0011$ & $-0,084^{* *}$ \\
\hline Verduras & $+4,48^{* *}$ & $+0,101^{* *}$ & $-0,014$ \\
\hline Legumes & $-0,62$ & $-0,194 * *$ & $-0,173^{* *}$ \\
\hline Frutas & $+3,28$ & $-0,29 * *$ & $+2,95^{* *}$ \\
\hline Fibras & $+0,11$ & $-0,146^{* *}$ & $+0,055$ \\
\hline Vitamina C & $+41,7^{* *}$ & $+1,08^{*}$ & $+5,80^{* *}$ \\
\hline Vitamina A & $+20,0$ & $-2,21 *$ & $-8,16^{* *}$ \\
\hline Ferro & $+1,27^{* *}$ & $-0,016^{* *}$ & $-0,011 *$ \\
\hline Sódio Total & $+180,3^{* *}$ & $-14,6^{* *}$ & $-26,1 * *$ \\
\hline Colesterol & $+19,0^{* *}$ & $-0,72^{* *}$ & $-1,85^{* *}$ \\
\hline
\end{tabular}

Nota: Dois asteriscos $(* *)$ indicam significância ao nível de 1\%; um $\left(^{*}\right)$ indica significância ao nível de 5\% e nenhum indica que a hipótese de nulidade do parâmetro não é rejeitada ao nível de significância de $5 \%$.

Por outro lado, controlando o efeito das características pessoais, a alimentação fora do domicilio apresenta menor teor de vitamina A e ferro.

De maneira geral, os resultados apresentados na Tabela 3 mostram que não é correto identificar a alimentação fora do domicilio com junk. food ou fast food. Há muita heterogeneidade na alimentação fora do domicilio, que inclui tanto o salgadinho do bar como a merenda escolar e o almoço na fábrica, produzido com orientação profissional. A alimentação no domicilio também é muito heterogênea, dependendo dos hábitos alimentares da pessoa e do seu grau de conscientização em relação à importância de uma alimentação saudável.

\section{Efeito de comer fora do domicílio no Índice de Massa Corporal (IMC), no sobrepeso e na obesidade}

Para analisar a relação entre comer ou não fora e o Índice de Massa Corporal (IMC) da pessoa, optouse por considerar apenas adultos, isto é, pessoas com 20 
ou mais anos de idade. Excluindo mulheres grávidas e pessoas com dados antropométricos biologicamente implausíveis, ficamos com uma amostra de 25.611 pessoas, incluindo 13.861 mulheres e 11.750 homens.

Em Hoffmann ${ }^{[10]}$ os dados da POF 2008-2009 são utilizados para analisar vários condicionantes do sobrepeso e da obesidade de adultos no Brasil, destacando-se os efeitos da escolaridade e da renda. Aos modelos utilizados naquele artigo foi acrescentada a variável "Come fora", que assume valor 1 se a pessoa eventualmente ingere alimentos fora do domicílio (conforme dados do inquérito alimentar) e assume valor 0 em caso contrário.

A Tabela 4 mostra a regressão múltipla estimada considerando o IMC das mulheres como variável dependente e os modelos de lógite para a probabilidade de uma mulher apresentar sobrepeso $\left(\mathrm{IMC} \geq 25 \mathrm{~kg} / \mathrm{m}^{2}\right.$ ) ou obesidade (IMC $\geq 30 \mathrm{~kg} / \mathrm{m}^{2}$ ).

Os coeficientes estimados mostram que "comer fora" tem uma relação negativa e estatisticamente significativa com o IMC de mulheres e com a probabilidade de uma mulher apresentar sobrepeso ou obesidade.

O efeito negativo é observado inclusive em uma análise sem o controle do efeito de outras variáveis. Entre mulheres que não comem fora do domicílio, 51,9\% apresentam sobrepeso, e para as que comem fora essa proporção é 42,6\%. O qui-quadrado da tabela de contingência 2 x 2 é fortemente significativo (probabilidade caudal inferior a 0,1\%).

Para obesidade, a proporção diminui de 19,6\% entre as mulheres que não comem fora para $14,4 \%$ entre as que comem fora. Novamente, uma associação negativa e fortemente significativa.

No caso dos homens, a proporção com sobrepeso aumenta de 50,0\% entre os que não comem fora para $52,0 \%$ entre os que comem fora. A associação é significativa ao nível de $5 \%$, mas não ao nível de $1 \%$. A proporção de obesos é $12,4 \%$ entre os que não comem fora e $11,6 \%$ entre os que comem fora, não havendo diferença estatisticamente significativa. Entretanto, estimando, para os homens, modelos como os apresentados na Tabela 4, o coeficiente para "comer fora" é negativo nas 3 equações (não-significativo a 5\% no modelo de lógite para sobrepeso mas significativo a 5\% no modelo de lógite para obesidade).
Observa-se, na Tabela 4, que o fato de se considerar preta aumenta significativamente o IMC e a probabilidade de a mulher apresentar sobrepeso ou obesidade. Curiosamente, como já foi constatado em Hoffmann [10], no caso dos homens esse efeito não existe ou tem, até mesmo, sinal contrário.

Aparentemente influenciados pelos trabalhos que mostram o efeito do fast food sobre o crescimento da obesidade, alguns pesquisadores tendem a assumir uma posição crítica em relação à alimentação fora do domicílio. Os resultados apresentados na Tabela 4 (e os resultados correspondentes para homens) indicam que "comer fora", no Brasil, está associado com menor probabilidade de a pessoa apresentar sobrepeso ou obesidade.

A interpretação do resultado tem necessariamente um caráter especulativo. Não nos parece que o coeficiente negativo reflita um efeito intrínseco ao "comer fora", mas sim o efeito de variáveis não controladas. O estilo de vida de uma pessoa está associado à atividade exercida; pode haver atividades que induzam, simultaneamente, a uma maior probabilidade de comer fora e maior cuidado com a alimentação (preocupação com a "silhueta"). Também não se pode descartar a existência de um efeito em sentido inverso, de obesidade sobre a probabilidade de comer fora do domicílio.

Analisando os dados da POF de 2002-2003, Bezerra \& Sichieri [11] verificam que, "no Brasil, o consumo de refeições fora do domicílio foi associado de maneira negativa com sobrepeso e obesidade entre mulheres, no entanto, apresentou associação positiva entre homens" (p. 228). É importante notar que nesse artigo a maneira de definir se uma pessoa "come fora do domicílio" ou não é baseada nas despesas com alimentação registradas pela pessoa durante uma semana. Nem seria possível usar a definição de "comer fora" adotada nos modelos da Tabela 4, pois na POF de 2002-2003 não foi feito o inquérito alimentar. Notese, por exemplo, que a ingestão da merenda escolar (que não implica em nenhuma despesa por parte do aluno) não é captada como "comer fora" nesse outro critério. Tendo em vista verificar se a diferença de resultados é devida à diferente definição de "comer fora", foi feita uma análise dos dados da POF de 20082009 considerando "comer fora" a existência de 
Tabela 4. Regressão múltipla para o Índice de Massa Corporal (IMC) e modelos de lógite para probabilidade de sobrepeso ou obesidade em mulheres com 20 ou mais anos de idade, no Brasil, em 2008-2009

\begin{tabular}{|c|c|c|c|c|c|}
\hline \multirow{2}{*}{\multicolumn{3}{|c|}{ Variável explanatória }} & \multirow{2}{*}{$\begin{array}{l}\text { Regressão múltipla } \\
\text { para o IMC }\end{array}$} & \multicolumn{2}{|c|}{ Modelo de lógite para } \\
\hline & & & & Sobrepeso & Obesidade \\
\hline \multicolumn{3}{|c|}{ Constante } & $16,898^{* *}$ & $-3,5982^{* *}$ & $-4,7792^{* *}$ \\
\hline \multicolumn{3}{|c|}{ Idade (em décadas) } & $2,888^{* *}$ & $1,1950^{* *}$ & $1,0557^{* *}$ \\
\hline \multicolumn{3}{|c|}{ Idade ao quadrado } & $-0,238^{* *}$ & $-0,0973^{* *}$ & $-0,0897 * *$ \\
\hline \multirow{7}{*}{\multicolumn{2}{|c|}{ Escolaridade: }} & $1-2$ & $0,620 * *$ & $0,2739 * *$ & $0,2539 *$ \\
\hline & & $3-4$ & $0,559 * *$ & 0,1326 & 0,1082 \\
\hline & & $5-8$ & 0,165 & 0,0876 & $-0,0398$ \\
\hline & & $9-10$ & $-0,042$ & $-0,0727$ & $-0,0193$ \\
\hline & & 11 & $-0,240$ & $-0,1399$ & $-0,2836^{* *}$ \\
\hline & & $12-14$ & $-1,352^{* *}$ & $-0,5850^{* *}$ & $-0,6629 * *$ \\
\hline & & $\geq 15$ & $-0,709^{* *}$ & $-0,4403^{* *}$ & $-0,2558$ \\
\hline \multicolumn{3}{|c|}{ Cor Preto } & $0,723 * *$ & $0,3086^{* *}$ & $0,3568^{* *}$ \\
\hline \multicolumn{3}{|c|}{ Pardo } & $0,211 *$ & 0,0545 & $0,1259 *$ \\
\hline \multicolumn{3}{|c|}{ Amarelo } & $-1,722^{* *}$ & $-0,6356^{* *}$ & $-0,1338$ \\
\hline \multicolumn{3}{|c|}{ Região Norte } & $0,475^{* *}$ & $0,1834 *$ & 0,1314 \\
\hline \multicolumn{3}{|c|}{$\mathrm{MG}+\mathrm{ES}+\mathrm{RJ}$} & 0,114 & $-0,0389$ & 0,1066 \\
\hline \multicolumn{3}{|c|}{ SP } & $0,789 * *$ & $0,1687 * *$ & $0,3761 * *$ \\
\hline \multicolumn{3}{|c|}{ Sul } & $0,823^{* *}$ & $0,2508^{* *}$ & $0,3804 * *$ \\
\hline \multicolumn{3}{|c|}{ Centro-Oeste } & 0,221 & 0,0173 & 0,0654 \\
\hline \multicolumn{6}{|c|}{ RFPC: } \\
\hline \multicolumn{3}{|c|}{ Mais de 140 a 280} & 0,171 & 0,1530 & 0,1251 \\
\hline \multicolumn{3}{|c|}{ Mais de 280 a 560} & 0,357 & $0,2210^{* *}$ & 0,1919 \\
\hline \multicolumn{3}{|c|}{ Mais de 560 a 840} & $0,564 * *$ & $0,3339 * *$ & $0,2751^{*}$ \\
\hline \multicolumn{3}{|c|}{ Mais de 840 a 1.120} & 0,413 & $0,2936^{* *}$ & $0,2646^{*}$ \\
\hline \multicolumn{3}{|c|}{ Mais de 1.120 a 1.400} & 0,306 & 0,2143 & 0,2175 \\
\hline \multicolumn{3}{|c|}{ Mais de 1.400 a 2.100} & $0,918^{* *}$ & $0,5483^{* *}$ & 0,2649 \\
\hline \multicolumn{3}{|c|}{ Mais de 2.100 a 3.500} & $0,729 * *$ & $0,2915^{*}$ & 0,2248 \\
\hline \multicolumn{3}{|c|}{ Mais de 3.500 a 5.600} & $-0,450$ & 0,0733 & $-0,2469$ \\
\hline \multicolumn{3}{|c|}{ Mais de 5.600} & 0,533 & 0,1359 & $0,7706^{* *}$ \\
\hline \multicolumn{3}{|c|}{ Urbano } & $0,493 * *$ & 0,0841 & $0,1598^{*}$ \\
\hline \multicolumn{3}{|c|}{ Metropolitano } & 0,059 & 0,0766 & 0,0318 \\
\hline \multicolumn{3}{|c|}{ Criança de 0 a 4 anos: 1} & $0,373^{* *}$ & $0,1629 * *$ & 0,1111 \\
\hline & & 2 ou mais & 0,451 & 0,1414 & $0,3995^{* *}$ \\
\hline
\end{tabular}

Continua 
Tabela 4. Continuação

\begin{tabular}{lc|c|c}
\hline \multirow{2}{*}{ Variável explanatória } & \multirow{2}{*}{$\begin{array}{c}\text { Regressão múltipla } \\
\text { para o IMC }\end{array}$} & \multicolumn{2}{|c}{ Modelo de lógite para } \\
\cline { 3 - 4 } & 0,079 & 0,0100 & Obesidade \\
\hline Criança de 5 a 9 anos: 1 & 0,324 & $0,2163^{*}$ & 0,1035 \\
2 ou mais & $-0,269^{* *}$ & $-0,0938^{*}$ & 0,0596 \\
Come fora do domićlio & 0,089 & - & $-0,1786^{* *}$ \\
Coeficiente de determinação & - & 0,656 & - \\
Medida de concordância $c$ & 13.861 & 13.861 & 0,635 \\
Número de observações & & & 13.861
\end{tabular}

Nota: Dois asteriscos $\left(^{* *}\right)$ indicam significância ao nível de $1 \%$; um $\left.*^{*}\right)$ indica significância ao nível de $5 \%$ e nenhum indica que a hipótese de nulidade do parâmetro não é rejeitada ao nível de significância de 5\%.

despesa semanal com alimentação fora do domicílio ${ }^{1}$. Para essa análise, como não são usados dados do inquérito alimentar, podemos utilizar uma amostra maior. Considerando pessoas com 20 ou mais anos de idade, exclusive indígenas, pessoas sem declaração de cor ou escolaridade, mulheres grávidas e aqueles com dados antropométricos implausíveis, ficamos com uma mostra de 118.964 observações (61.347 mulheres e 57.617 homens).

Para tornar o texto mais conciso, vamos usar "despesa fora" para indicar a existência de despesa semanal com alimentação fora do domicílio.

Considerando as 26.056 pessoas da amostra para as quais dispomos dos valores das variáveis binárias "comer fora" e "despesa fora", verifica-se que a associação entre as duas está longe de ser perfeita. Mais de um terço $(38,3 \%)$ das pessoas que comem fora não têm "despesa fora" e 26,8\% daquelas sem registro de comer fora no primeiro dia do inquérito alimentar têm "despesa fora".

Utilizando a amostra de 118.964 pessoas, verifica-se que aquelas com "despesa fora" tem RFPC média maior ( $\mathrm{R} \$ 1.265,5$ versus $\mathrm{R} \$ 753,2$ para os sem

\footnotetext{
${ }^{1}$ A despesa com alimentação fora do domicílio está sempre no Registro Tipo 12 - Despesa individual, podendo ser associada a uma pessoa. As despesas com alimentação no domicilio estão no Registro Tipo 11 - Caderneta de despesa (por Unidade de Consumo).

${ }^{2} \mathrm{O}$ coeficiente de associação $\varphi$ é igual a 0,34 .
}

"despesa fora"), escolaridade média maior $(9,0$ versus 6,4 anos) e idade média menor (40,4 versus 45,9 anos).

Entre mulheres sem "despesa fora", 50,0\% apresentam sobrepeso e essa proporção se reduz a $45,1 \%$ nas demais. A proporção de obesas diminui de $17,8 \%$ entre as sem "despesa fora" para 15,5\% entre as com "despesa fora". Nos dois casos a associação é altamente significativa. Em modelos de lógite para sobrepeso e obesidade, com os mesmos controles considerados na Tabela 4, o coeficiente de uma variável binária para "despesa fora" é negativo, mas claramente não significativo.

Para homens a proporção com sobrepeso aumenta de $47,3 \%$ entre os sem "despesa fora" para $52,8 \%$ entre os com "despesa fora". A proporção de obesos aumenta de $11,6 \%$ para $13,1 \%$. Nos dois casos a associação é estatisticamente significativa e com sinal oposto à observada entre mulheres. Em modelos de lógite para sobrepeso e obesidade de homens, com os mesmos controles considerados na Tabela 4, o coeficiente da variável binária para "despesa fora" é positivo, sendo não significativo no modelo de lógite para obesidade, mas significativo a 1\% no modelo para sobrepeso. No caso dos homens há uma forte associação positiva entre "despesa fora" e maior IMC, mesmo procurando controlar estatisticamente os efeitos de renda, escolaridade etc., confirmando-se o resultado de Bezerra \& Sichieri [11]. Tendo em vista os resultados apresentados anteriormente, isso não permite concluir que a causa do sobrepeso seja o fato de comer fora do domicílio. Usando os dados do 
inquérito alimentar não foi possível constatar efeito positivo de "comer fora" na probabilidade de um homem apresentar sobrepeso. Há forte efeito positivo da renda sobre a probabilidade de um homem ter sobrepeso [10] e é muito difícil, por meio de modelos de regressão múltipla ou lógite, isolar o efeito de "comer fora" (ou ter "despesa fora").

O fato de o efeito de comer fora (ou de ter "despesa fora") ser negativo para mulheres mostra que as pessoas não estão "condenadas" a ingerir a alimentação oferecida que pode gerar sobrepeso e obesidade. Decisões pessoais são fundamentais. Obviamente, o reconhecimento desse fato não implica em reduzir a importância da regulamentação da propaganda de alimentos e do papel das leis e dos órgãos públicos na promoção de uma alimentação saudável.

\section{CONCLUSÃO}

O grupo de pessoas que eventualmente come fora do domicilio, no Brasil, é muito diferente do grupo dos que não o fazem. A renda familiar per capita média dos primeiros é 45\% maior. Assim, comparar a dieta desses dois grupos de pessoas pode levar a conclusões errôneas sobre o efeito de comer fora na qualidade da alimentação ingerida. Analisando os dados referentes a pessoas que comem tanto dentro como fora do domicílio, é possível comparar a composição dos dois tipos de alimentação controlando os efeitos de todas as características pessoais. Os resultados não permitem concluir que a alimentação fora do domicílio seja de pior qualidade. Embora seu teor de açúcar seja maior, seu teor de lipídeos é menor e seu teor de vitamina C é maior do que na alimentação ingerida no domicílio. No Brasil, não é correto identificar a alimentação fora do domicílio com ingestão de fast food.

Verificou-se, ainda, que, quando se controla cuidadosamente o efeito de idade, escolaridade, renda, etc., o efeito de comer fora sobre o IMC e sobre a probabilidade de uma mulher apresentar sobrepeso ou obesidade é negativo. No caso dos homens esse efeito também é negativo, embora estatisticamente menos significativo do que no caso das mulheres, e se torna positivo quando, em lugar da variável "comer fora", se considera a existência de despesa com alimentação fora do domicílio durante uma semana.

\section{REFERÊNCIAS}

[1] Bezerra IN. Impacto do consumo de alimentos fora do domicílio na dieta e no peso corporal da população brasileira (tese). Rio de Janeiro: Universidade do Estado do Rio de Janeiro, Faculdade de Ciências Médicas; 2012. $178 \mathrm{p}$.

[2] Bezerra IN, Souza AM, Pereira RA, Sichieri R. Contribution of foods consumed away from home to energy intake in Brazilian urban areas: the 2008-9. Nationwide Dietary Survey. Br J Nutr. 2013;109(7):127683.

[3] Gorgulho BM. Alimentação fora do lar e sua relação com a qualidade da dieta de moradores do município de São Paulo: estudo ISA-Capital (dissertação). Universidade de São Paulo, Faculdade de Saúde Pública; 2012. 92 p.

[4] Bezerra IN, Souza AM, Pereira RA, Sichieri R. Consumo de alimentos fora do domicílio no Brasil. Rev Saúde Públ. 2013;47(Supl. 1):200-11.

[5] Abreu ES, Garbelotti ML, Torres EAFS. Dietary fiber consumption and composition of foods in "by-the-kilo" restaurants. Nutrition \& Food Science. 2005;35(6):386-92.

[6] Rodrigues AGM, Proença RPC, Calvo MCM, Fiates GMR. Perfil da escolha alimentar de arroz e feijão na alimentação fora de casa em restaurante de bufê por peso. Ciênc Saúde Colet. 2013;18(2):335-46.

[7] Instituto Brasileiro de Geografia e Estatística (IBGE). Pesquisa de Orçamentos Familiares 2008-2009: análise do consumo alimentar pessoal no Brasil. Rio de Janeiro: IBGE; 2011.

[8] Instituto Brasileiro de Geografia e Estatística (IBGE). Pesquisa de Orçamentos Familiares 2008-2009: tabelas de composição nutricional dos alimentos consumidos no Brasil. Rio de Janeiro: IBGE; 2011.

[9] Hoffmann R. Determinantes do consumo da merenda escolar no Brasil: análise dos dados da PNAD de 2004 e 2006. Seg Alim Nutr. 2012;19(1):33-45.

[10] Hoffmann R. Condicionantes do sobrepeso e da obesidade de adultos no Brasil, 2008-2009. Seg Alim Nutr. 2012;19 (2):1-16.

[11] Bezerra IN, Sichieri R. Características e gastos com alimentação fora do domić́lio no Brasil. Rev Saúde Públ. 2010;44(2):221-29. 